\title{
Is Sputtering Sufficient for Production of Replicas?
}

\author{
Nadezda Vaskovicova, Kamila Hrubanova and Vladislav Krzyzanek
}

Institute of Scientific Instruments of the CAS, v.v.i., Brno, Czech Republic.

In the production of replicas there are many parameters which influence the authenticity and precision of the biological structures, e.g. manipulation with tissue pieces or cells, conditions of freeze-fracture technique or the process of replica preparation alone. The original protocols of replica processing have employed for many years devices such as BAF060 (BAL-TEC) that is equipped by cryo-stage and two guns for well specified directional metal and carbon evaporation [1]. This technology is relatively expensive, in comparison to modern devices for cryo-preparation for electron microscopy like ACE600 (Leica microsystems). However, these modern devices are usually based on the metal sputtering in the argon atmosphere and evaporation of carbon (e.g. ACE600 or the precursor MED020). Here we report on the protocol for production replicas using both types of devices (ACE600 and BAF060) and comparison of the results.

The investigations were performed on the tissue of mouse stomach epithelium; pieces of the tissue were fixed by $3 \%$ glutaraldehyde, washed in PBS buffer and saturated by $20 \%$ glycerol in the fridge overnight. Such fixed samples were placed on the freeze-fracture carriers and frozen in melting nitrogen. In the case of processing in the ACE600 device the frozen samples on the carriers were moved at sample holder into ACE600 by the shuttle VCT100 (Leica microsystems). Consequently, the samples were fractured and etched at $-100^{\circ} \mathrm{C}$ for $13 \mathrm{~min}$, the surface was sputtered by platinum of $2.1 \mathrm{~nm}$ at the angle $50^{\circ}$ in the argon atmosphere of the pressure $1 \times 10^{-3}$ mbar, and, finally, a carbon layer of $21 \mathrm{~nm}$ was evaporated at the angle $90^{\circ}$ in pressure of $1 \times 10^{-6}$ mbar.

Next series of samples were processed by BAF060 in the standard way: the frozen tissues were fractured and etched at $-100^{\circ} \mathrm{C}$ for $10 \mathrm{~min}$. Then, the fractured structures were covered by evaporation of the platinum of $2.4 \mathrm{~nm}$ at the angle $50^{\circ}$ (pressure $1 \times 10^{-6}$ mbar) followed by evaporation of carbon of $22.4 \mathrm{~nm}$ at the angle $90^{\circ}$ (pressure $1 \times 10^{-5}$ mbar).

After these steps all samples were melted at the room temperature and the tissue remnants were removed by sulfuric acid and sodium hypochlorite from the metal replicas. The replicas were viewed in the TEM Morgagni 268D (FEI).

The quality of replicas is comparable for both, the ACE600 and the BAF060 devices, up to the magnification of 56000 . This magnification corresponds to observation of the structure of organelles, Figure 1A, B. The ultrastructure of membranes shows same distribution of intramembrane particles (Figure 1 C, D). However, there are some discrepancies on the higher magnification (mag. 89 000) in the replicas made in ACE600; e.g. the deposits of platinum in the form of separated dots (white arrows in Figure 1C). So far we cannot conclude if this is artefact caused by platinum sputtering in argon atmosphere, or by using relatively lower vacuum in range $10^{-3} \mathrm{mbar}$, or using not pure argon. That artefact can have a little influence on the interpretation of the morphological structures and also on a quantitative analysis of the image.

Although there is a fundamental difference in the methodological process of applying the platinum in the ACE and the BAF devices, we found results of the replica texture comparable. For the purpose of preservation, the fractured biological structures are replicas processed in the ACE device sufficient. 


\section{References:}

[1] JE Heuser, Journal of electron microscopy 60 (2011), p. 3.

[2] The research was supported by MEYS CR (LO1212) and its infrastructure by MEYS CR and EC (CZ.1.05/2.1.00/01.0017).
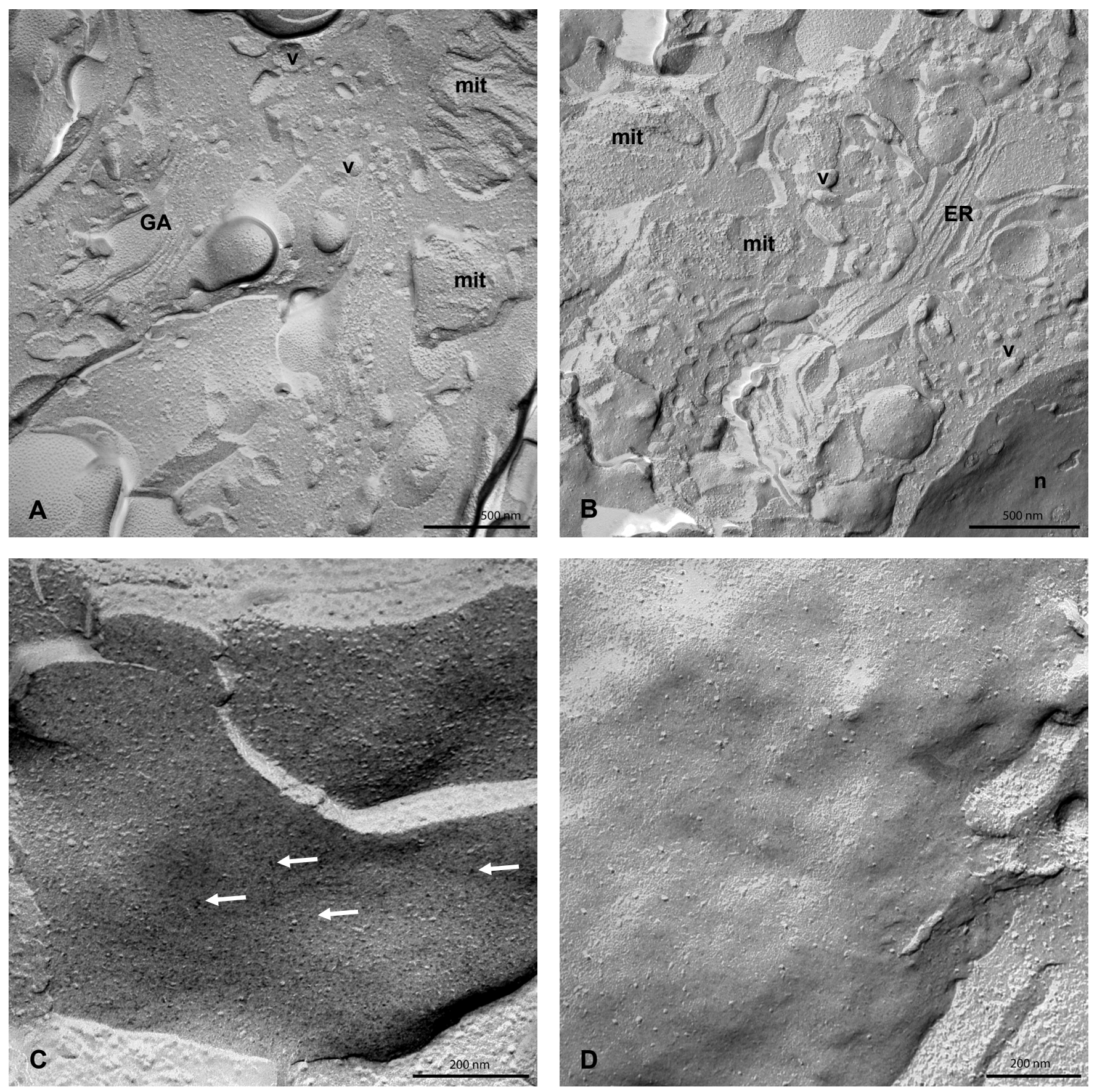

Figure 1. A, B replicas of the intracellular spaces with the organelles; C, D replicas of the plasma membranes. The A, $\mathbf{C}$ replica made in ACE600 device; the B, D replica made in BAF060 device; mit mitochondrion, GA Golgi apparatus, ER endoplasmic reticulum, $\mathbf{n}$ nucleus, white arrows sputtering artefacts formed by platinum. 